\section{Adaptación rápida a un modelo de docencia virtual: estudio de caso en la asignatura Didáctica de la Geometría}

\section{Steven Van Vaerenbergh}

Universidad de Cantabria

\section{Resumen}

Este artículo describe el proceso docente experimentado en una asignatura universitaria ante la situación inédita provocada por la COVID-19. Se analiza en detalle el proceso de adaptación de la docencia a un formato no presencial al inicio del periodo de confinamiento en marzo de 2020, cuando prácticamente de un día para otro hubo que cambiar la enseñanza presencial en enseñanza virtual y plantear un enfoque personalizado y activo. En este caso, durante los primeros días se recogieron datos y opiniones de los estudiantes mediante un cuestionario que permitieron adecuar la docencia no presencial a todos los estudiantes. Esta contribución recoge, en primer lugar, los resultados de un cuestionario inicial, en los cuales se basaron las modificaciones seguidas para la docencia no presencial. A continuación, se describe el modelo adoptado, los cambios en la evaluación y algunas experiencias durante el trascurso de la docencia. Se analiza además la efectividad del modelo seguido mediante los resultados de un cuestionario de seguimiento que se pasó al terminar la docencia y la evaluación. Finalmente, se ofrece una serie de conclusiones y reflexiones sobre esta experiencia.

Palabras clave: métodos de enseñanza, docencia virtual, docencia no presencial, docencia universitaria, tecnología educativa
Quick adaptation to a virtual teaching model: Case study in the subject Didactics of Geometry

\section{Abstract}

This article describes the teaching process experienced in a university subject in the face of the unprecedented crisis caused by the COVID-19. It analyses in detail the process of adapting teaching to a non-attendance format at the beginning of the confinement period in March 2020, when practically overnight a change from attendance-based teaching to virtual teaching was required, following a personalised and active approach. In this case, during the first days, data and student opinions were collected by means of a questionnaire to allow for the adaptation of distance teaching to all students. This contribution firstly includes the results of an initial questionnaire, on which the modifications followed for distance teaching were based. Next, it describes the model adopted, the changes in evaluation and some experiences during the course of teaching. The effectiveness of the model followed is also analysed through the results of a follow-up questionnaire that was passed on when teaching and evaluation were completed. Finally, a series of conclusions and reflections on this experience are offered.

Keywords: teaching methods, virtual teaching, distance learning, university teaching, educational technology

\section{Introducción}

La crisis sanitaria actual causada por la COVID-19 ha afectado a la educación de más de 1.500 millones de estudiantes a nivel mundial. Según las cifras de la UNESCO (UNESCO, 2020), en su reunión del 31 de marzo de 2020, casi el $91 \%$ de los estudiantes del mundo, matriculados en 188 países, han estado confinados en sus casas y sin clases presenciales. En España, solamente en la Enseñanza Superior más de 300 mil estudiantes se han visto afectados. Esta situación llegó de forma inesperada. Las universidades y los centros educativos y formativos centraron sus esfuerzos en asegurar la continuidad del aprendizaje, haciendo lo menos brusco posible el cambio de metodología en los procesos de enseñanza para generalizar el uso de medios digitales y de docencia no presencial. Y dentro de ella, la de una

\title{
MAGISTER
}

Vol. 32. Núm. I: (2020). Sección extraordinaria 
enseñanza digital. Profesores y alumnos, con o sin experiencia previa en formación virtual, se han visto inmersos necesariamente en esta dinámica.

El 16 de marzo de 2020 se suspendieron las actividades docentes presenciales en la Universidad de Cantabria, al igual que en las demás universidades españolas. En la asignatura Didáctica de la Geometría del segundo curso del Grado en Educación Primaria de la Facultad de Educación, en ese momento, había 244 alumnos matriculados. Los alumnos estaban divididos en cuatro grupos y cada grupo tenía su propio profesor. En este artículo se detallan los cambios en la docencia de uno de esos grupos, el grupo B, que contaba con un total de 68 alumnos y que tenían clases presenciales 5 horas a la semana, divididas en tres sesiones.

\section{Primera semana}

Puesto que faltó tiempo para estudiar la forma más eficaz de impartir la docencia a distancia, el primer día se trató de continuar con las clases de teoría y ejercicios usando un medio virtual. Para ello, se usó la plataforma de videoconferencia Skype Profesional ${ }^{1}$ de la que la propia universidad ya contaba con la correspondiente licencia (ver Figura 1). La asistencia era de más de 50 alumnos, lo cual era comparable con el número de asistentes a las clases presenciales. Aunque la conexión en general era buena, enseguida se notaron algunas limitaciones de este tipo de docencia. En particular, los estudiantes no tenían una forma fácil para pedir la palabra y, como consecuencia, simplemente usaban el chat para escribir sus preguntas, dudas y respuestas a las preguntas del profesor. Como estaba claro que en una situación tan improvista no todos los alumnos iban a contar con los medios o la disponibilidad para asistir a esta clase, el profesor grabó esta clase y la puso disponible en una carpeta virtual donde pondría además disponibles todas las grabaciones de las sesiones siguientes durante el resto del curso.

${ }^{1}$ https://www.skype.com/es/business/ Skype Profesional de Microsoft.

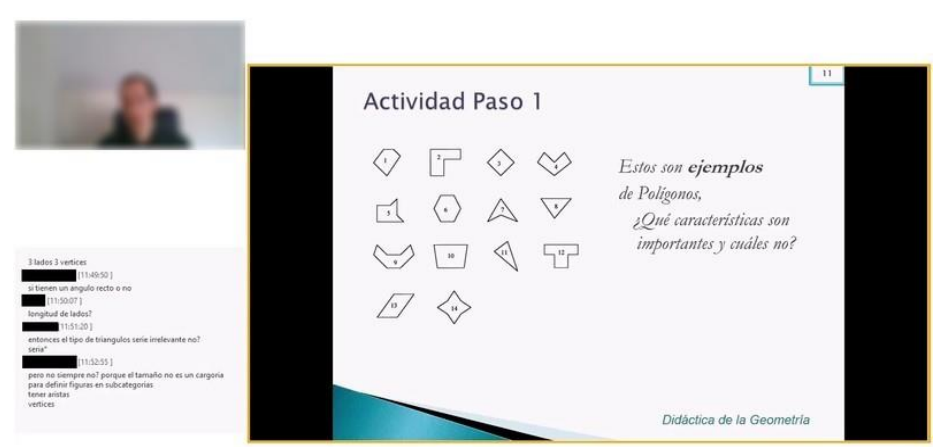

Figura 1. Captura de pantalla de una videoconferencia con la pantalla del profesor (derecha), la imagen del profesor y el chat.

El segundo día, se continuó impartiendo la clase de esta forma, aunque ese día ya se notaban mayores problemas de conexión, tanto por parte del profesor como de los alumnos, quizás debido a la congestión de la red causada por un mayor número de personas trabajando y estudiando desde casa. Como en este momento ya había quedado claro que simplemente intentar replicar las clases presenciales en un formato online usando una herramienta de videoconferencia no iba a ser una opción adecuada para la mayoría de los estudiantes, se decidió pasar un cuestionario para recoger las experiencias de los estudiantes hasta ese momento y para pedir opiniones y sugerencias. Los resultados de este cuestionario inicial se presentan a continuación.

El tercer y último día de clase de esa semana se hizo una sesión de ejercicios todavía siguiendo el guion de la docencia presencial. Aunque en esta sesión se exploró brevemente la posibilidad de usar una tableta gráfica como pizarra virtual para resolver los ejercicios, resultó mucho más útil para este fin el software GeoGebra² GeoGebra es un popular programa de matemática dinámica, que ya formaba parte de las clases habituales en esta asignatura (ver Figura 2). Entre otros, se usa para ilustrar conceptos geométricos con dibujos y permite demostrar visualmente que algunas propiedades de una construcción se mantienen, aunque se arrastren partes de una figura. Usando la funcionalidad de compartir pantalla, este software resultó adecuado como pizarra virtual para las clases a distancia.

${ }^{2}$ https://www.geogebra.org/ GeoGebra.

\section{MAGISTER}

Vol. 32. Núm. I: (2020). Sección extraordinaria 


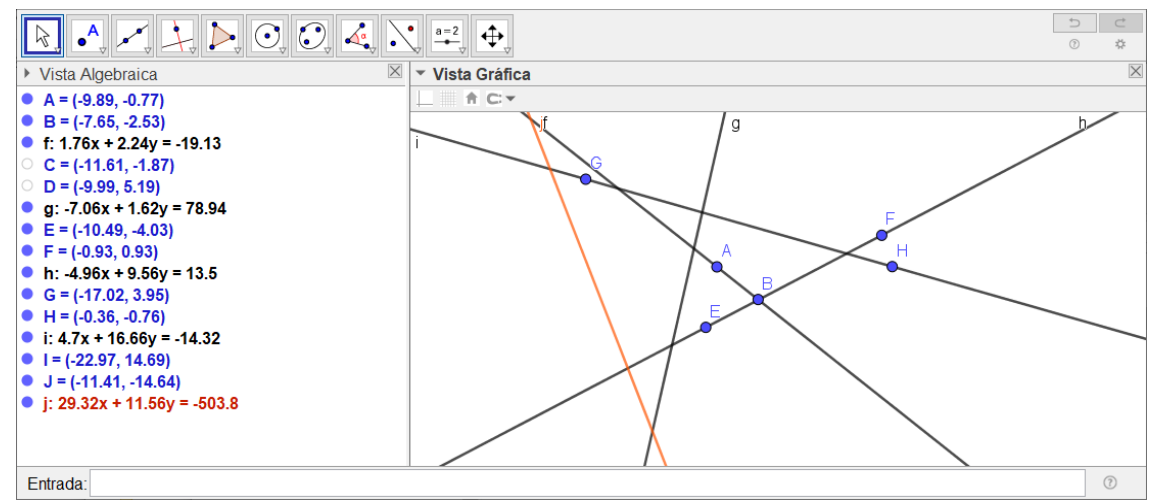

Figura 2. Uso de GeoGebra como pizarra virtual.

\section{Cuestionario inicial}

Después del segundo día de clase a distancia se pasó un cuestionario breve para conocer las experiencias y las sugerencias de los estudiantes. Las respuestas recogidas corresponden a $n=42$ estudiantes. En primer lugar, se preguntó sobre la calidad de la conexión durante las primeras dos sesiones. Los resultados están recogidos en la Figura 3. Como se puede observar, hubo grandes diferencias en la calidad de la conexión, la imagen y el audio.

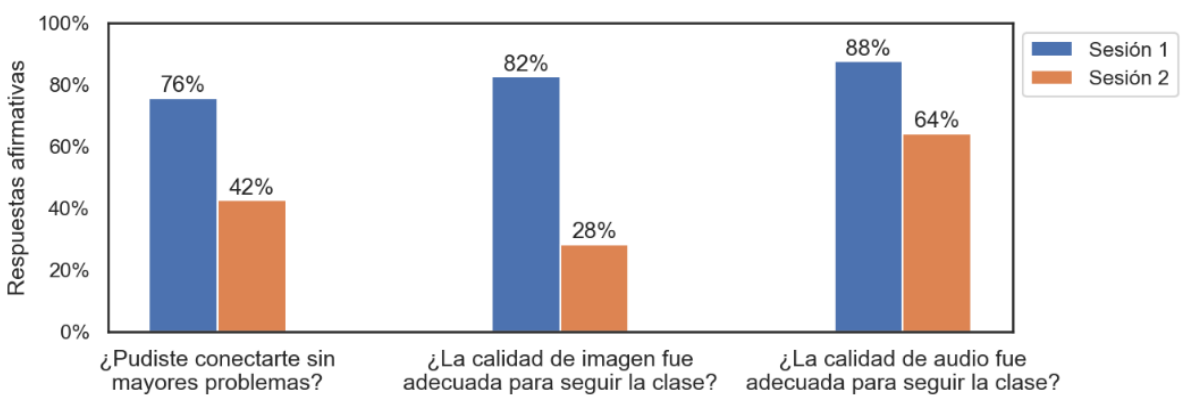

Figura 3. Opiniones sobre la calidad de la conexión, la imagen y el audio de la videoconferencia durante la primera y segunda sesión.
A continuación, se preguntó cuál sería, en la opinión de los estudiantes, un formato adecuado para seguir las clases de teoría a distancia. De los 42 participantes, un 38\% opinó que las videoconferencias le parecían un formato adecuado si la conexión era buena. Un 50\% prefería que el profesor preparase vídeos de la materia y que los colgase online para que cada alumno pudiera verlos a su ritmo, como se ve en la Figura 4.

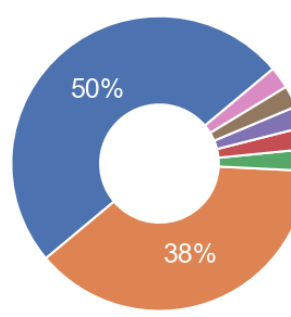

¿Qué formato te parece bueno para las clases de teoría? - Prefiero ver la teoría en un vídeo preparado por el profesor. El formato actual (videoconferencia Skype), si la conexión es buena.

Figura 4. Preferencias de los alumnos sobre el formato de las clases de teoría a distancia Las opciones con menos respuestas se detallan en el texto principal.

Esta pregunta también permitía sugerencias, visibles como las demás opciones en la Figura 4, entre las cuales se encontraban las ideas de explorar otras plataformas de videoconferencia, el estudio individual a base de diapositivas o apuntes y usar un formato mixto de videos, hechos por el profesor, con sesiones de videoconferencias para dudas.

La siguiente pregunta del cuestionario trataba específicamente la modalidad de las clases de tutoría, visualizada en la Figura 5. En este caso, la mayoría de los estudiantes (el 68\% de las respuestas) prefería hacer tutorías grupales mediante videoconferencia, contrario al $32 \%$ que prefería hacer tutorías por email. No se ofrecieron más opciones para contestar y 2 participantes se abstuvieron.

\section{MAGISTER}

Val. 32. Núm. I: (2020). Sección extraordinaria 


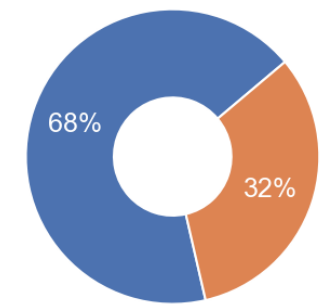

¿Cómo te gustaría hacer las sesiones de tutoría grupal? Por teleconferencia.

Por email.

Figura 5. Preferencias de los alumnos sobre el formato de las clases de tutoría grupal a distancia.

El cuestionario también preguntaba sobre la modalidad preferida para las clases de ejercicios y las prácticas con ordenador, que se imparten con GeoGebra. Las respuestas a esta pregunta fueron muy variadas, desde la sugerencia de hacerlo individualmente con unas instrucciones más detalladas hasta la opción de posponer las actividades hasta que se retomase la docencia presencial.

\section{Modelo adoptado}

Basado en los datos y las opiniones recogidos en este cuestionario, se decidió adoptar una modalidad virtual-mixta de docencia para el grupo B: El profesor grabaría vídeos para explicar la parte teórica de la asignatura, y los colgaría en una plataforma en la nube, en este caso YouTube ${ }^{3}$. Los estudiantes dedicarían 2 de las 5 horas semanales de clase a ver y estudiar estos vídeos. Durante las otras 3 horas se harían tutorías grupales a través de la herramienta de videoconferencia que ya se había empezado a usar. Para evitar problemas de conexión se pasaría a una configuración que usase algo menos de ancho de banda, entre otros, limitando la imagen a diapositivas y pantallas virtuales. Las grabaciones de estas tutorías se colgarían en la nube (privada) de la universidad. Para todos los documentos de texto de la asignatura, como diapositivas, hojas de ejercicios y artículos académicos, ya se estaba usando la instalación de la plataforma Moodle ${ }^{4}$ de la universidad. Las clases de prácticas interactivas de GeoGebra se sustituirían por tareas individuales

${ }^{3}$ https://www.youtube.com/ YouTube.

MAGISTER

Val. 32. Núm. I: (2020). Sección extraordinaria usando unas instrucciones más detalladas que las que se usaban en las sesiones presenciales.

\section{Cambios en la evaluación de la asignatura}

En condiciones normales, la evaluación de la asignatura consistía en un $60 \%$ de la nota obtenida en el examen (presencial) y el resto se correspondía a tareas de evaluación continua y resultados de las sesiones prácticas. En el mes de abril, cuando se hizo evidente que no había posibilidad de llevar a cabo un examen presencial, se decidió extender la parte correspondiente a la evaluación continua mediante un trabajo adicional y reemplazar el examen presencial por un cuestionario en Moodle para evaluar el conocimiento teórico, que contaba un $40 \%$ de la nota final. El cuestionario en Moodle consistía en 2 partes, cada una con 16 preguntas, que en su totalidad cubrían todos los contenidos de la asignatura. Las preguntas eran breves y los estudiantes disponían de 15 minutos para realizar cada parte. Para evitar que dos estudiantes recibieran las mismas preguntas, se creó un banco de preguntas con un elevado número de preguntas y se usaron varias opciones de aleatorizar el examen.

De los 244 estudiantes matriculados en toda la asignatura, 233 participaron en el examen en Moodle, y este trascurrió sin problemas de conexión ni de otro tipo.

\section{Cuestionario de seguimiento}

Al finalizar la docencia y las evaluaciones se pasó un cuestionario de seguimiento al grupo B para conocer la efectividad y la satisfacción de la modalidad de docencia y evaluación seguida. Participaron $n=28$ estudiantes y la mayoría (93\%) reconoció haber seguido las clases de teoría en YouTube. Véase Figura 6.

\footnotetext{
${ }^{4}$ https://moodle.org/ Moodle.
} 


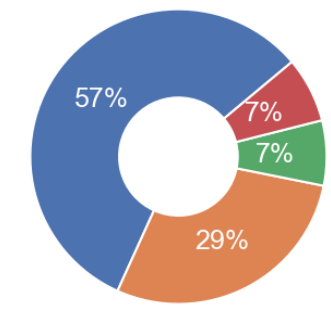

¿Has visto las clases de teoría en YouTube? Sí, todas.
Sí, la mayoría.
Sí, unas pocas

Figura 6. Porcentajes de estudiantes que han seguido las clases de teoría en YouTube.

Los vídeos de teoría se publicaban antes de las sesiones de tutoría grupal correspondientes para que los estudiantes pudieron estudiar la teoría y preparar sus dudas. Sin embargo, no todos los estudiantes aprovecharon esta posibilidad, tal y como muestra la Figura 7.

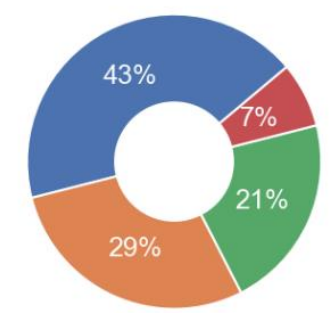

¿Has visto las clases de teoria en YouTube antes o después de la tutoria grupal correspondiente?

En general antes.

A veces antes y a veces después.

En general después.

No he visto las clases de teoría en YouTube.

Figura 7. Momento en que los estudiantes han visto los vídeos de contenido teórico.

Las videoconferencias con las tutorías grupales fueron aprovechadas por el $100 \%$ de los encuestados, como se puede observar en la Figura 8. El 57\% de los participantes confirma haber seguido la clase sobre todo en vivo, mientras que el $14 \%$ optaba por ver las grabaciones.

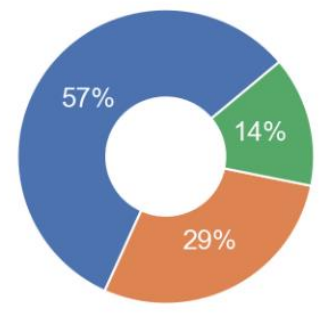

¿Seguiste las clases de tutoria grupal? Si, sobre todo por Skype.

$\mathrm{Si}$, algunas veces por Skype y otras viendo las grabaciones. $\mathrm{Si}$, sobre todo viendo las grabaciones colgadas.
Figura 8. Modalidades en las cuales los estudiantes han seguido las tutorías grupales.

El cuestionario también preguntaba sobre la calidad de la conexión, la imagen y el audio durante el modelo de docencia virtual adoptado, tal como se hizo en el cuestionario inicial. Los resultados se pueden ver en la Figura 9, que incluye los resultados iniciales de la Figura 3 para facilitar la comparación. Se puede observar que los problemas de conexión quedaron principalmente resueltos.

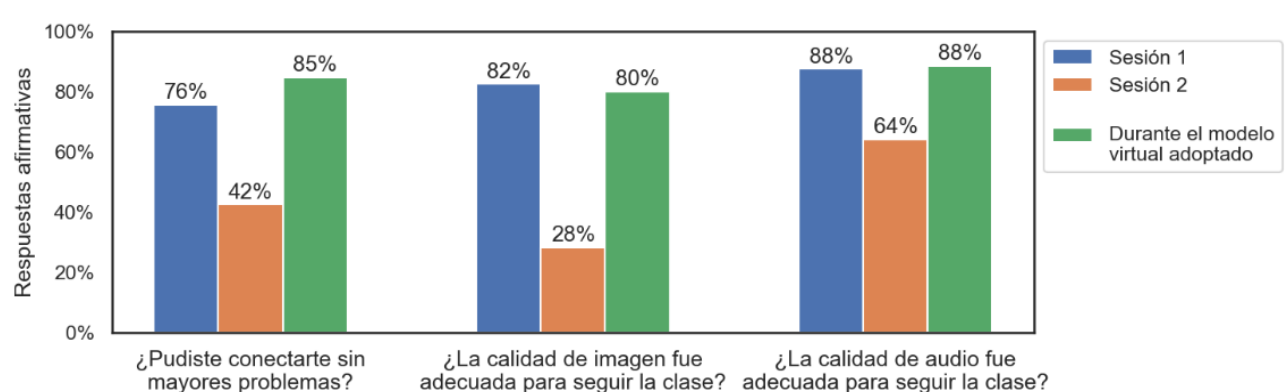

Figura 9. Opiniones sobre la calidad de la conexión, la imagen y el audio de la videoconferencia en las primeras dos sesiones, y durante la modalidad de docencia virtual adoptada.

Ante la pregunta "Las sesiones de GeoGebra se hicieron de forma individual usando una hoja de pautas adicionales. ¿Te ha parecido adecuado este formato online?" el $86 \%$ contestó que sí y el $14 \%$ contestó que no. Un resultado similar se recogió para la pregunta " ¿El cuestionario en Moodle te ha parecido un formato adecuado para la evaluación de los conocimientos teóricos?”: El 86\% contestó que sí y el 14\% que no.

\section{MAGISTER}

Vol. 32. Núm. I: (2020). Sección extraordinaria 
En cuanto a la forma de consultar sus dudas, el $64 \%$ reconoció haber consultado al profesor y el 71\% ha consultado a otro estudiante, tal y como muestra la Figura 10.

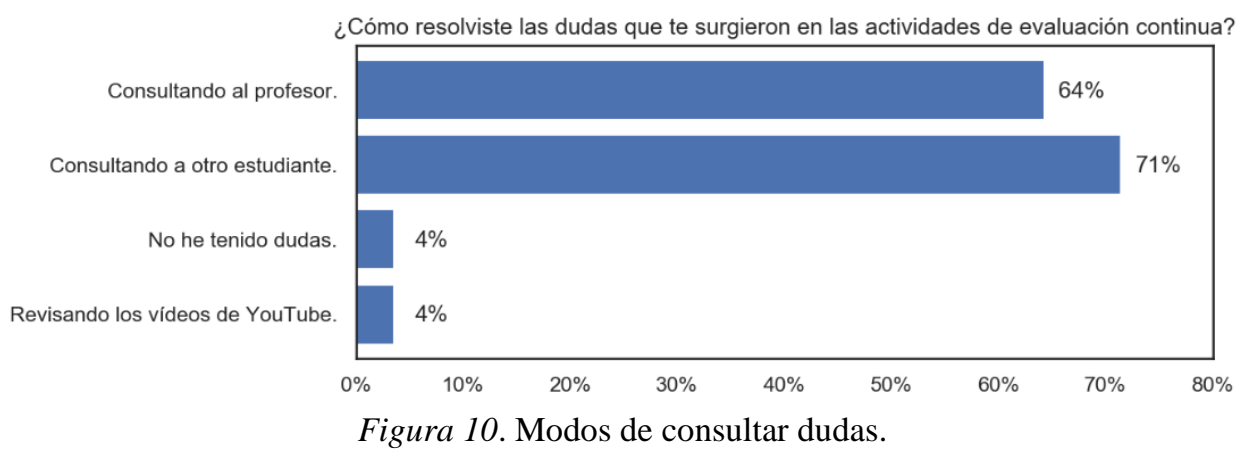

Finalmente, se pidió que los estudiantes valorasen el tiempo disponible para realizar el examen en Moodle, que consistía en aproximadamente 1 minuto por pregunta, teniendo en cuenta que las preguntas eran muy cortas. Como se puede observar en la Figura 11, a la mayoría de los encuestados (14, o el 50\%) les pareció un tiempo adecuado, mientras que un $32 \%$ ( 9 encuestados) indicó que le faltaba tiempo y el $19 \%$ (5 encuestados) dijo que le sobraba tiempo.

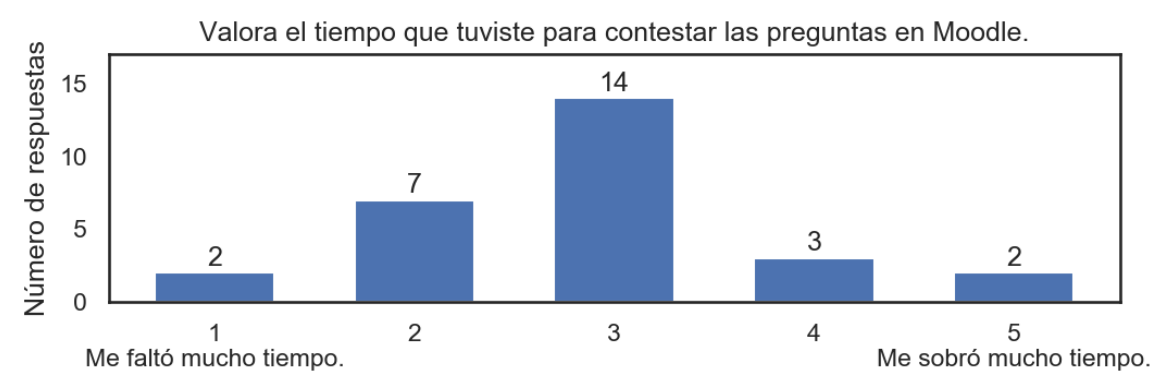

Figura 11. Valoración del tiempo disponible para el examen en Moodle.
En los comentarios recogidos algunos estudiantes indicaron que el tiempo disponible era insuficiente para reflexionar en profundidad sobre algunas preguntas planteadas, aunque reconocieron que en general el formato era acertado.

\section{Conclusiones}

La educación superior se enfrenta a importantes desafíos por la pandemia de la COVID-19 que seguramente influirán en su futuro. La organización de la docencia resulta especialmente afectada en este contexto. En este artículo se ha detallado el proceso de adaptar la docencia de una asignatura universitaria a una modalidad virtual de forma rápida e imprevista debido al confinamiento por la crisis sanitaria actual. La situación que se presentó no tenía antecedentes y por ello, tras una primera exploración de posibles modalidades de docencia virtual, se decidió recoger datos y opiniones de los estudiantes para diseñar un modelo viable para todos. El modelo que se adoptó combinaba vídeos grabados por el profesor con sesiones de videoconferencia para las tutorías grupales. Una vez implantado en la segunda semana del confinamiento, se mantuvo este formato hasta el final. También se realizó un cambio hacía una modalidad de evaluación más basada en evaluaciones continuas. Aunque quedaron al descubierto algunas deficiencias del modelo de docencia adoptado, como pueden ser la limitada interactividad en las herramientas elegidas, en general, este modelo ha cubierto la mayor parte de las necesidades de docencia y la respuesta del alumnado ha sido muy positiva.

\section{Referencias}

UNESCO (2020). Impacto de Covid-19 en la Educación - Datos. Paris, Francia: UNESCO. UNESCO. Recuperado el 22/07/2020 desde https://es.unesco.org/covid19/educationresponse

*Autor de contacto: Steven Van Vaerenbergh, steven.vanvaerenbergh@unican.es

\section{MAGISTER}

Vol. 32. Núm. I: (2020). Sección extraordinaria 